Josef Riedmann (éd.), Die Innsbrucker Briefsammlung. Eine neue Quelle zur Geschichte Kaiser Friedrichs II. und König Konrads IV.

Benoît Grévin

\title{
OpenEdition
}

Journals

Édition électronique

URL : http://journals.openedition.org/ifha/9041

DOI : 10.4000/ifha. 9041

ISSN : 2198-8943

Éditeur

IFRA - Institut franco-allemand (sciences historiques et sociales)

Référence électronique

Benoît Grévin, « Josef Riedmann (éd.), Die Innsbrucker Briefsammlung. Eine neue Quelle zur Geschichte Kaiser Friedrichs II. und König Konrads IV. », Revue de I'IFHA [En ligne], Date de recension, mis en ligne le 23 mai 2018, consulté le 24 septembre 2020. URL : http://journals.openedition.org/ifha/9041 ; DOI : https://doi.org/10.4000/ifha.9041

Ce document a été généré automatiquement le 24 septembre 2020.

(CIFHA 


\section{Josef Riedmann (éd.), Die Innsbrucker Briefsammlung. Eine neue Quelle zur Geschichte Kaiser Friedrichs II. und König Konrads IV.}

Benoît Grévin

\section{RÉFÉRENCE}

Josef Riedmann (éd.), Die Innsbrucker Briefsammlung. Eine neue Quelle zur Geschichte Kaiser Friedrichs II. und König Konrads IV., Wiesbaden: Harrassowitz Verlag, 2017, 334 p., $80 €$ 
L'édition de la collection de lettres d'Innsbruck, très attendue par les spécialistes, mérite d'être présentée au lectorat francophone à travers un grand nombre de canaux. La découverte en 2004 par Josef Riedmann, à l'occasion d'un travail de catalogage des manuscrits médiévaux de la Universitäts- und Landesbibliothek Tirol in Innsbruck, d'un ensemble de 148 textes inédits (mandats et lettres) émanant des chancelleries de l'empereur Frédéric II et de son fils Conrad IV lors de son court règne effectif en Sicile (1252-1254), dans une collection de 209 textes, est un événement majeur de la recherche sur l'histoire de la Sicile, de l'Empire et des derniers Hohenstaufen. Il a reçu une relativement large publicité en

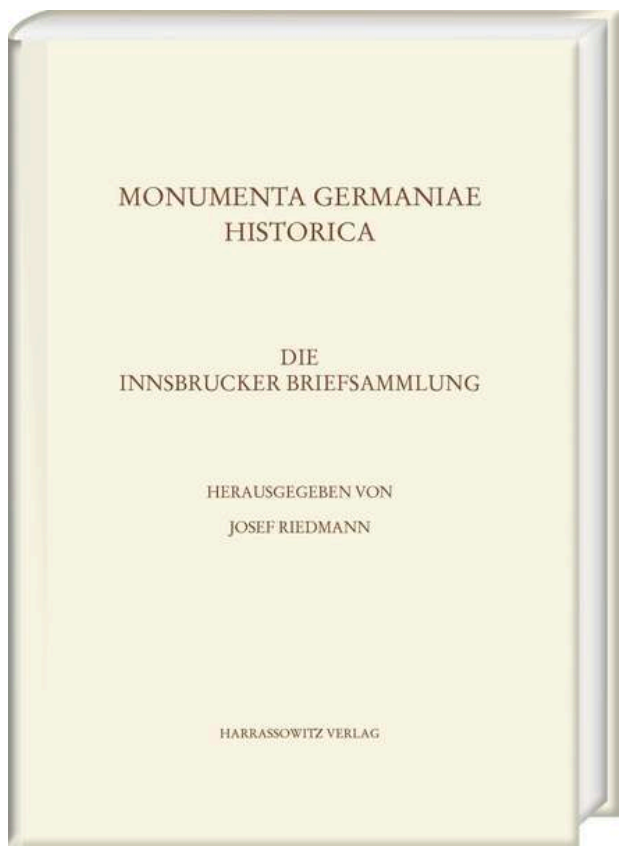
Allemagne, une publicité plus réduite en Italie, mais est passé quasiment inaperçu en France. La parution, douze ans plus tard, en 2017, d'une édition de ces lettres et mandats dans la collection « Briefe des späteren Mittelalters » des MGH vient donc combler une attente entretenue par la publication de divers articles (notamment de la plume de J. Riedmann) qui ont donné un aperçu de la richesse de cette documentation sans l'épuiser. C'est J. Riedmann lui-même qui, aidé par divers spécialistes, a mené à bien la tâche difficile d'interpréter ces documents, transmis dans un manuscrit riche en abréviations et posant des problèmes de lecture de tous ordres.

Ajoutons que cette superbe collection - la découverte d'une série de lettres royales inédites inconnues du XIII ${ }^{\mathrm{e}}$ siècle en si grand nombre est un fait exceptionnel présente des problèmes d'interprétation d'autant plus considérables que, pour une bonne part d'entre eux, les textes n'ont pas été préservés intégralement. Selon la logique caractéristique des summae dictaminis issues de la cour de Sicile (pour simplifier, des collections de «lettres de Pierre de la Vigne ", à laquelle notre collection peut être en partie assimilée dans la mesure où elle présente un certain nombre de textes présents dans la version la plus classique des lettres $\mathrm{PdV}$ ), les textes ont ici été sélectionnés pour servir de modèle d'écriture rhétorique en chancellerie. Bien souvent, les salutationes, les noms de personnes, les protocoles ont été abrégés, voire supprimés, et de nombreuses lettres ont été copiées seulement partiellement.

Comme maintes éditions princeps, ce volume présente des textes sous un aspect qui pourra parfois être encore amélioré. Certaines leçons ont parfois été transcrites telles qu'elles, alors qu'elles ne peuvent pas avoir été écrites sous cette forme par les notaires responsables de cette documentation. D'autre part, la recherche des sources utilisées par les rédacteurs pourra être poussée plus à fond, notamment en ce qui concerne les classiques. Nous ne discuterons pas ici de ces points strictement philologiques mais importants, dans la mesure où l'art de l'écriture politique au XIII ${ }^{\mathrm{e}}$ siècle, l'ars dictaminis, est intimement lié à un ensemble de connaissances rhétoriques et à des techniques de rédaction que nous qualifierions aujourd'hui de partiellement littéraires. On trouvera 
quelques éléments dans un volume prochain de Francia, dans l'attente d'une discussion plus approfondie qui nécessitera un plus large espace. Certaines interprétations historiques de la documentation devront également être discutées. On se contentera ici, en soulignant que les aspects perfectibles de l'édition de J. Riedmann ne remettent pas en cause son intérêt global, de présenter rapidement les points saillants de cette documentation.

Les 148 lettres inédites contenues dans ce volume se divisent entre une minorité de 36 lettres remontant au règne de Frédéric II (et plutôt, pour celles qui peuvent être datées avec certitude, à sa dernière partie), et une majorité de 112 lettres datables du séjour en Sicile de Conrad IV, descendu d'Allemagne pour prendre le contrôle du regnum en 1252, et qui mourut après l'avoir pacifié et réorganisé au printemps 1254, alors qu'il lançait une expédition pour restaurer les bases du parti pro-Staufen en Italie du Nord. Ce déséquilibre suggère déjà l'intérêt réel de la collection. Les 36 lettres " frédériciennes ", précieuses mais souvent d'interprétation difficile, ne modifient qu'à la marge notre connaissance du règne de Frédéric II, pour lequel nous disposons de milliers de documents semblables. Elles permettent tout de même de confirmer certains points déjà connus, par exemple concernant les rapports de l'empereur avec ses deux bâtards Enzio et Frédéric d'Antioche, ses actions politiques en Italie centrale (Tivoli) et en Lombardie, ses relations avec des princes de l'Empire, dans l'espace italien (Savoie) ou Outre-Alpes (ducs de Lorraine). En revanche, même si le nom de Conrad IV séduit moins le grand public et n'exerce pas le même pouvoir de fascination intrinsèque, principalement à cause du caractère avorté de son règne, les 112 documents inédits le concernant modifient très sensiblement notre connaissance de son activité, d'autant plus que nous ne disposions pas jusqu'ici d'une série documentaire d'une très grande richesse.

Pour les années 1252-1254, la collection se révèle donc de tout premier ordre et jette effectivement une vive lumière sur des aspects de l'activité royale dont nous ignorions à peu près tout. C'est par exemple le cas de la réorganisation du royaume à travers la création (l'Aquila, pour laquelle le rôle majeur de Conrad IV est confirmé) ou la réorganisation des villes (amélioration du port de Salerne, de celui de Barletta). C'est aussi, et peut-être surtout, le cas de la diplomatie du nouveau roi, qui cherche visiblement au début de son règne sicilien effectif à tisser la toile d'un vaste réseau reprenant la tradition paternelle. Le dossier formé par les lettres diplomatiques adressées aux souverains et au principaux pouvoirs européens est à cet égard d'une richesse extrême, s'étendant de la Navarre et de la France à Nicée et à la Hongrie, en passant par la papauté (avec des documents prouvant le rôle d'honnête courtier de la Sicile entre Nicée et la papauté, malgré l'excommunication des Hohenstaufen!) ou encore par la Sardaigne (avec la reprise de contact avec Adelaide de Torres, femme du demi-frère de Conrad, Enzio, emprisonné à Bologne). Tout cela ne forme qu'une partie d'un ensemble extrêmement complexe, qui comprend également des lettres de consolation (litterae plangentium, "Trostenbriefe»), à mi-chemin entre l'exercice rhétorique et la réitération politique d'une amitié dynastique ou d'un lien avec des familiares.

Pour exploiter comme elles le méritent ces nouvelles sources, il faudra donc à la fois prolonger le travail d'analyse politique et factuelle déjà entrepris par J. Riedmann et d'autres chercheurs, et entreprendre une sérieuse étude de leurs aspects rhétoriques, qui permettra peut-être d'en dire plus sur l'identité du notaire de Conrad IV qui a 
probablement porté cette collection dans l'espace alpin dès la mort du roi, isolant ainsi ces pièces de la tradition qui a abouti à la création des versions classiques des Lettres de Pierre de la Vigne. Les lettres inédites de la collection d'Innsbruck ne sont pas seulement une preuve spectaculaire qu'il est encore possible, en ce début de XXI ${ }^{e}$ siècle, de retrouver une documentation politique de première importance remontant au XIII ${ }^{\mathrm{e}}$ siècle dans les bibliothèques d'Europe, et que les opérations de catalogage peuvent réserver de grandes surprises. Elles sont un maillon essentiel pour reconstituer une histoire politique qui, pour se terminer en impasse, n'en prolonge pas moins de manière passionnante celle de Frédéric II et pour améliorer notre connaissance d'un art d'écrire que la chancellerie sicilienne, en ce milieu du XIII ${ }^{e}$ siècle, avait porté à son apogée $\mathrm{l}$ l'ars dictaminis.

INDEX

Index chronologique : Moyen Âge

Thèmes : Sources, Histoire des États et des pouvoirs

\section{AUTEUR}

BENOÎT GRÉVIN

CNRS-LAMOP 